\title{
A pilot eConsultation service in Eastern Ontario: bridging clinical genetics and primary care
}

\author{
Priya T. Bhola ${ }^{1}$ - Clare Liddy ${ }^{2,3} \cdot$ Amir Afkham $^{4} \cdot$ Erin Keely ${ }^{5,6,7} \cdot$ Gail E. Graham ${ }^{1,8}$
}

Received: 6 September 2018 / Revised: 4 December 2018 / Accepted: 5 January 2019 / Published online: 18 February 2019

(c) European Society of Human Genetics 2019

\begin{abstract}
With the rising demand for clinical genetics services, it is a challenge for clinical geneticists to meet the needs of patients and referring primary care providers in a timely way, using current models of genetics health care delivery. One method of providing primary care providers with greater access to clinical genetics expertise is through an electronic consultation (eConsult) service. We describe here a pilot project of a clinical genetics eConsult service that our genetics centre in Eastern

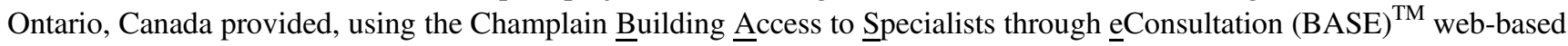
application. We analyzed 111 genetics eConsults submitted by primary care providers to a single clinical geneticist over a 28 -month time period. More than half (54\%) of the eConsult questions were regarding (1) hereditary cancer and (2) genetic syndromes, with the remainder encompassing a wide variety of clinical genetics topics. We avoided a referral to the Genetics clinic for an in-person appointment for $30 \%$ of the eConsult cases, based on a contemplated referral rate to Genetics clinic of $72 \%$ prior to eConsult and a planned referral rate to Genetics clinic of $42 \%$ following all eConsults. Primary care providers rated the eConsult service of high value to themselves and also to their patients. This pilot service supports the potential of an eConsultation service to create a stronger and more dynamic link between clinical genetics and primary care providers, which may lead to better patient care.
\end{abstract}

Priya T. Bhola

pbhola@cheo.on.ca

1 Department of Genetics, Children's Hospital of Eastern Ontario, Ottawa, Ontario, Canada

2 CT Lamont Primary Healthcare Research Centre, Bruyere Research Institute, Ottawa, Ontario, Canada

3 Department of Family Medicine, University of Ottawa, Ottawa, Ontario, Canada

4 The Champlain Local Health Integration Network, Ottawa, Ontario, Canada

5 Department of Medicine, University of Ottawa, Ottawa, Ontario, Canada

6 Division of Endocrinology/Metabolism, The Ottawa Hospital, Ottawa, Ontario, Canada

7 Ottawa Research Institute, Ottawa, Ontario, Canada

8 Department of Pediatrics, University of Ottawa, Ottawa, Ontario, Canada

\section{Introduction}

There has been a significant increase in the demand for clinical genetics services that will continue for the foreseeable future. This is in part due to the rapid pace of gene discovery and laboratory technological advances, which have produced a rising availability of clinical genetic tests. Other contributing factors include the discovery of new single gene conditions with management implications, such as hereditary cancer predisposition syndromes, and also increased evidence of a substantial genetic basis for conditions such as non-syndromic autism spectrum disorder, thus resulting in more indications for a Genetics consultation. Genetic test results are no longer binary and often require specific expertise for interpretation; this has resulted in increased referrals to Genetics clinics and an increased consultation complexity associated with follow-up appointments. As more genetic conditions are definitively diagnosed, there is also a concomitant cascade testing of family members. Meeting the needs of patients and referring primary care providers (PCPs) in a timely way is a challenge for geneticists using current models of genetics health care delivery, particularly in our Canadian 
publicly funded health care system where resources have not grown at the rate of service demand.

Technological applications that have enhanced genetics health care delivery have included genetic counseling via telephone and videoconferencing [1-3], as well as email consultations between specialists and patients about specific clinical situations [4]. Emerging models of genetics health care delivery include (1) multidisciplinary specialist teams such as the integration of genetic counsellors into oncology clinics $[5,6]$ and (2) the integration of genetics and genomics into primary care [7]. With genetic testing increasingly available to PCPs, timely support from geneticists is more important than it has been in the past. Already, PCPs ordering routine investigations such as chromosomal microarray would like greater access to geneticists [8], and recognize that they will need even more access to geneticists with the advent of whole genome sequencing [9].

Electronic consultations, defined as asynchronous, consultative, provider-to-provider communication within a secure web-based platform [10], have been described as efficient and effective patient care for a variety of subspecialties [11, 12]. To date, there have been no reports describing experience with a genetics eConsult service. Within the Champlain Local Health Integration Network (LHIN), one of 14 regional health regions in the province of Ontario, Canada, that serves a population of 1.4 million, the

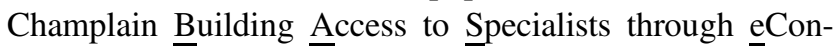
sultation $(\overline{\mathrm{BASE}})^{\mathrm{TM}}$ service has demonstrated improved access to many sub-specialties to date [13-18]. The success of this and other programs has led to the planned development of a provincial eConsult program [19]. Our centre, the Regional Genetics Program at the Children's Hospital for Eastern Ontario (CHEO), is the sole provider of all types of genetics services within the Champlain LHIN, including prenatal and hereditary cancer services, with the exception of care for inborn errors of metabolism, which is provided by a separate department. There are 1500-2000 new referrals to the Regional Genetics Program at CHEO per year from family doctors and sub-specialists, and a total of 5000-6000 visits with clinical geneticists and genetic counsellors, including follow-up appointments. Urgent referrals, such as prenatal cases, are often seen within days; however, wait times for elective indications can exceed 12 months depending on the type of referral and provider availability. Physicians outside Genetics have access to genetic testing; investigations ordered by other physicians include but are not limited to chromosomal microarray and Fragile X testing indicated for global developmental delay or intellectual disability, and karyotypes indicated for aneuploidy or infertility. Some specialty providers order specific sequencing panels-such as those for epilepsy or hearing loss. These tests are most often ordered by pediatricians, pediatric sub-specialists and fertility specialists and not PCPs. In our region PCPs order non-invasive prenatal testing for pregnant patients, but do not otherwise commonly order genetic testing. We have a geneticist on-call 24 hours per day and also a prenatal genetic counsellor oncall during weekday hours. Other resources for PCPs are online through our institution's website and the Genetics Education Canada Knowledge Organization (GECKO) website.

Our objective was to assess the feasibility of the Champlain BASE ${ }^{\mathrm{TM}}$ eConsult service for genetics eConsults over a 28-month time period, as well as evaluate referral outcomes and value as perceived by PCPs.

\section{Methods}

We used the previously described [20, 21] Champlain BASE $^{\mathrm{TM}}$ eConsult system, an asynchronous, secure, webbased application that facilitates the submission of patientspecific clinical questions via a standardized electronic form used by PCPs (typically family doctors and nurse practitioners). There were no inclusion or exclusion criteria regarding submission of an eConsult question. PCPs were able to attach additional information, such as laboratory results and digital images, to their eConsult question. The specialist was notified of an eConsult through email, which contained a link to the eConsult platform and she accessed the confidential platform using a username and password. The specialist response options included providing recommendations, requesting additional information or recommending a referral for an in-person clinical genetics appointment. The eConsult service was brought to the attention of PCPs by the Champlain BASE ${ }^{\mathrm{TM}}$ eConsult group through small group presentations at local practices, larger presentations at Continuing Medical Education events and newsletters. The service began as a pilot project with organic growth. There was not a systemic capture of all local providers. A turnaround time of 1 week from submitting a question to receiving an initial response was an expectation for the Champlain $\mathrm{BASE}^{\mathrm{TM}}$ eConsult service. The specialists received reminders about outstanding eConsults before the 1-week deadline. The geneticist and the PCP had ongoing access to closed eConsults, and the platform allowed for the entirety of an eConsult exchange to be printed.

From 27 November 2013 to 29 March 2016, 114 genetics eConsults were submitted to the Champlain $\mathrm{BASE}^{\mathrm{TM}}$ eConsult system. All were completed by one geneticist at our centre (GG), who was compensated on the same pro-rated time-based scale used for all types of specialist physicians providing eConsult services within the Champlain LHIN. Data were prospectively collected, securely stored and then retrospectively accessed for 
analysis. The data collected from each eConsult included provider type, age and gender of patient, and result from a mandatory close-out survey completed by the PCP. In most cases, the length of time from receiving the eConsult email link to responding to the eConsult question as estimated by the specialist was available.

We retrospectively categorized genetics eConsults into one of seven clinical categories: hereditary cancer, genetic syndrome, connective tissue disorder, hemoglobinopathy, metabolic condition, isolated congenital anomaly or other. We further sub-categorized two categories: hereditary cancer (sub-categorized into: hereditary breast and ovarian cancer, gastro-intestinal and other cancer) and genetic syndrome (sub-categorized into: chromosomal, dysmorphic features, intellectual disability, skeletal dysplasia, congenital anomalies and other). A preliminary list of categories was reached by consensus and following categorization of the first 25 eConsults; a finalized list of categories was again reached by consensus. After the final analysis, the clinical category 'other' was noted to be a large group (28/111), and further sub-categorized. We also categorized each eConsult by the type of clinical question being asked (prenatal screening, diagnosis of a genetic condition or management sub-categories).

The Champlain BASE ${ }^{\mathrm{TM}}$ eConsultation platform includes a mandatory close-out survey consisting of five questions completed by the PCP at the conclusion of each eConsultation. Question 1 was 'Which of the following best describes the outcome of this eConsult for your patient?' with the following options: (1) I was able to confirm a course of action that I originally had in mind, (2) I got good advice for a new or additional course of action, (3) I did not find the response very useful and (4) None of the above (please comment). Question 2 was regarding referrals, and asked PCPs to select one of the following: (1) Referral was originally contemplated but now avoided at this stage, (2) Referral was originally contemplated and is still neededthis eConsult likely leads to a more effective visit, (3) Referral was not originally contemplated and is still not needed - this eConsult provided useful feedback/information, (4) Referral was not originally contemplated but eConsult process resulted in a referral being initiated, (5) There was no particular benefit to using eConsult in this case and (6) Other (please comment). Question 3 asked PCPs to rate the overall value of the eConsult service for their case for the patient on a scale of 1 (minimal value) to 5 (excellent value). Question 4 asked PCPs to rate the overall value of the eConsult service for their case for themselves as a PCP on a scale of 1 (minimal value) to 5 (excellent value). Question 5 was an open-ended question that allowed for any additional feedback to be free texted.

We obtained research ethics board approval from The Ottawa Health Science Network Research Institute.

\section{Results}

There were 114 genetics eConsults submitted from 27 November 2013 to 29 March 2016 by 76 PCPs. One hundred seven of the 114 eConsults were submitted by a physician and seven were submitted by a nurse practitioner. During this time period, 52 of 76 providers (68\%) submitted one eConsult question and 24 of 76 providers (32\%) submitted between two and five questions.

Three of the 114 submitted eConsults were excluded from the analysis: two concerned the management of clinical disorders appropriate for another specialty (polycystic ovarian syndrome and antiphospholipid syndrome) and one concerned paternity testing for a non-medical indication that was out of scope of clinical genetics services. Nineteen of the $111(17 \%)$ analyzed eConsults concerned pediatric patients and 92/111 (83\%) concerned adult patients. The geneticist's time to complete the eConsult was self-reported for 94 of 114 eConsults as: $7 \%$ completed in less than 10 minutes, $49 \%$ completed in 10-15 minutes, $29 \%$ completed in 15-20 minutes and 15\% completed in $20+$ minutes. The clinical geneticist typically responded to submitted eConsults within 2 business days. There were occasional instances of a single back and forth between the geneticist and PCP, which usually occurred over a 1-2 day period.

We assigned a clinical category (e.g. type of genetic condition) and a type of clinical question (screening, diagnosis and management) for each eConsult (Table 1), with further sub-categorization (Table 2). The most common clinical category was hereditary cancer $(33 \%)$, with just over half of the questions (19/37) relating to hereditary breast and ovarian cancer. The second most common clinical category was genetic syndrome $(21 \%)$, with just over one-third of questions (8/23) relating to chromosomal conditions. The 'other' category included neurogenetics (5 eConsults) as well as 12 diverse genetic conditions, which were each the topic of an eConsult between one and three times (Table 2). We did not receive any eConsults regarding direct to consumer testing.

The close-out survey requested that PCPs retrospectively disclose whether they intended to refer their patient for an inperson appointment prior to the eConsult, and whether that intention was altered by the geneticist's eConsult response (Table 1). Of the 80/111 patients for whom a referral to Genetics was contemplated prior to the eConsult, the PCP response suggested that a referral was avoided in $38 / 80$ (47.5\%) and still planned in $42 / 80$ (52.5\%). Of the $28 / 111$ patients for whom a referral to Genetics was not contemplated prior to the eConsult, the PCP response suggested that a referral would be initiated for 5/28 (18\%) and still avoided for the remaining 23/28 (82\%). Overall then, an inperson referral was contemplated for $72 \%$ of patients before eConsultation and only $42 \%$ following eConsultation, 
Table 1 Clinical categories of eConsults and referral outcomes

\begin{tabular}{|c|c|c|c|c|c|}
\hline \multirow[t]{2}{*}{ Clinical Category } & \multirow{2}{*}{$\begin{array}{l}\text { Number of } \\
\text { eConsults } \\
(\%)\end{array}$} & \multicolumn{4}{|c|}{ Referral intent before eConsult and outcomes following eConsult } \\
\hline & & $\begin{array}{l}\text { Referral } \\
\text { contemplated } \\
\text { and initiated }\end{array}$ & $\begin{array}{l}\text { Referral } \\
\text { contemplated } \\
\text { and avoided }\end{array}$ & $\begin{array}{l}\text { Referral not } \\
\text { contemplated, } \\
\text { still avoided }\end{array}$ & $\begin{array}{l}\text { Referral not } \\
\text { contemplated, } \\
\text { newly initiated }\end{array}$ \\
\hline Hereditary cancer & $37(33 \%)$ & 16 & 12 & 7 & 1 \\
\hline Genetics syndrome & $23(21 \%)$ & 13 & 6 & 3 & 1 \\
\hline Connective tissue & $6(5 \%)$ & 2 & 0 & 2 & 1 \\
\hline Hemoglobinopathy & $4(4 \%)$ & 1 & 3 & 0 & 0 \\
\hline Metabolics & $7(6 \%)$ & 3 & 2 & 2 & 0 \\
\hline $\begin{array}{l}\text { Isolated congenital } \\
\text { anomaly }\end{array}$ & $6(5 \%)$ & 1 & 1 & 3 & 1 \\
\hline Other & $28(25 \%)$ & 6 & 14 & 6 & 1 \\
\hline \multirow[t]{3}{*}{ Total $(\%)$} & 111 & $42(38 \%)$ & $38(34 \%)$ & $23(21 \%)$ & $5(5 \%)$ \\
\hline & & \multicolumn{4}{|c|}{ Total contemplated referrals (prior to eConsult): $72 \%$} \\
\hline & & \multicolumn{4}{|c|}{ Total planned referrals (following eConsult): $42 \%$} \\
\hline
\end{tabular}

suggesting that eConsultation potentially allowed $30 \%$ of referrals to be avoided. The clinical categories in which a contemplated referral was most often avoided were hereditary cancer (12/37 eConsults) and 'other' (14/28 eConsults). The five eConsult cases in which no referral was contemplated with an outcome of a referral newly planned were from five different clinical categories. For 3/111 eConsults, the PCP indicated that the referral outcome was none of the options listed above; for two of these cases there were comments that a new eConsult about a different question would be considered, pending further discussion with the patient.

The most frequent type of clinical question asked in an eConsult concerned the management of a genetic condition with management of a genetic family history being the most common sub-category (Table 3 ). The next most frequent type of clinical question concerned the diagnosis of a genetic condition (24/111 eConsults) or prenatal screening (10/111 eConsults). On the survey following completion of each eConsult, PCPs reported that the eConsult service was of high value to themselves and to their patients (Fig. 1). For 66/111 (59\%) of eConsults, the PCP reported receiving good advice for a new or additional course of action. For 17/111 (15\%) of eConsults, the PCP included additional written feedback; these were all positive comments, mostly on educational value, helpfulness of the eConsult to a specific case and comments on the prompt response time.

\section{Discussion}

This is the first instance of an eConsult service used in a clinical genetics setting to our knowledge. This study demonstrated that an eConsultation service for genetics is feasible, has the potential to significantly reduce unnecessary referrals and is of value to PCPs. We found that approximately half of the eConsults submitted (54\%) concerned a hereditary cancer or genetic syndrome and the remainder concerned a highly diverse spectrum of clinical topics (Tables 1 and 2). These disorders were also asked about in various settings, ranging from prenatal screening to diagnosis to management. This breadth of diverse clinical disorders and the various settings in which they might arise highlights the increasing role of genetics in primary care, the challenges that genetic issues pose to PCPs and the value of closer, timely communication between PCPs and geneticists.

Notably, we potentially avoided a referral to the genetics clinic (for an in-person appointment) for 30\% of eConsults, comparable to the $28-35 \%$ described by other specialities who have used the Champlain BASE ${ }^{\mathrm{TM}}$ eConsult service [13-18]. Although some of these avoided referrals may have been declined or redirected at the referrals triage stage, avoiding this step saves professional and support staff resources in both offices, reduces frustration and provides the PCP with some guidance and confidence in his or her course of action. In cases when a referral was deemed necessary, eConsultation allowed the specialist to advise the PCP to gather additional information or order additional investigations to make the best use of waiting time and enhance the specialist visit when it occurred. We demonstrated a high level of provider satisfaction, specific to genetics eConsults. More than $85 \%$ of PCPs rated the eConsult service as being of excellent value for themselves and their patients, which is comparable to what has been described by other specialties who have used the Champlain BASE $^{\mathrm{TM}}$ eConsult service, and also with the $70-95 \%$ of providers reporting high satisfaction in other studies [10]. In 
Table 2 Sub-categorization of cancer, syndromic and other clinical categories

\begin{tabular}{|c|c|c|c|}
\hline \multirow{2}{*}{$\begin{array}{l}\begin{array}{l}\text { Clinical } \\
\text { category }\end{array} \\
\begin{array}{l}\text { Hereditary } \\
\text { cancer }\end{array}\end{array}$} & \multirow{2}{*}{$\begin{array}{l}\begin{array}{l}\text { Number of } \\
\text { eConsults }\end{array} \\
37 / 111\end{array}$} & \multicolumn{2}{|l|}{$\begin{array}{l}\text { Sub-category of clinical disorder } \\
\text { and number of eConsults }\end{array}$} \\
\hline & & HBOC & 19 \\
\hline & & GI & . \\
\hline & & Cancer-other & 11 \\
\hline \multirow{6}{*}{$\begin{array}{l}\text { Genetics } \\
\text { syndrome }\end{array}$} & $23 / 111$ & Chromosomal & 8 \\
\hline & & Dysmorphism & \\
\hline & & ID & 2 \\
\hline & & Skeletal dysplasia & \\
\hline & & Congenital anomalies & \\
\hline & & Syndrome-Other & \\
\hline \multirow[t]{13}{*}{ Other } & $28 / 111$ & Neurogenetics & 5 \\
\hline & & Alpha-1 antitrypsin deficiency & \\
\hline & & Cystic fibrosis & \\
\hline & & MTHFR & 2 \\
\hline & & Thrombophilia & 2 \\
\hline & & $\begin{array}{l}\text { Pseudocholinesterase } \\
\text { deficiency }\end{array}$ & 2 \\
\hline & & $\begin{array}{l}\text { NT interpretation and } \\
\text { management }\end{array}$ & 2 \\
\hline & & Fever syndrome & 2 \\
\hline & & Malignant hyperthermia & \\
\hline & & Cardiogenetics & 1 \\
\hline & & Spina bifida occulta & 1 \\
\hline & & ADPKD & 1 \\
\hline & & Hemochromatosis & 1 \\
\hline
\end{tabular}

Table 3 eConsults and type of clinical question

\begin{tabular}{lc}
\hline Type of clinical question & Number of eConsults \\
\hline Prenatal screening & 10 \\
Diagnosis of a genetic condition & 24 \\
Management & 61 \\
Family history & 11 \\
Known condition & 5 \\
Interpretation of test result & \\
Total & 111 \\
\hline
\end{tabular}

terms of the time demands on the geneticist, $85 \%$ of eConsults, for which the time to complete the consult was recorded, were completed in 15-20 minutes or less, with $56 \%$ of eConsults being completed in $10-15$ minutes or less. Our general specialist time for completion of an eConsult was longer than what has previously been reported by a pediatric eConsult study [14]; however, this is not unexpected given the greater diversity of genetics consults, the rarity of genetic conditions and the frequent need to consult the medical literature and other online resources. New in-person genetics assessments are typically 1 hour in duration, thus the relatively short amount of time to complete an eConsult compares favourably with the potential clinic time saved.

This was a pilot study of 114 eConsults directed to Genetics and completed by one geneticist at our centre, with prospective collection of data and retrospective analysis. A strength of our study is that we were able to use a previously piloted web-based application, the Champlain $\mathrm{BASE}^{\mathrm{TM}}$ eConsult system. With a robust and confidential platform, there is the potential to distribute the eConsults to geneticists in different locations serving the same region. An eConsult platform is also generalizable to different specialties. Limitations of our study include the relatively small number of eConsults analysed and our inability to follow-up on long-term outcomes, such as the number of referrals made to genetics clinic following an eConsult (versus the number of planned referrals), and the number of cases that would have been declined at triage. Studies with larger numbers of genetics eConsults that track long-term outcomes may be helpful in implementing an eConsult service into clinical practice. Although we did not obtain a patient perspective, it has been reported that patients whose PCP used an eConsult service on their behalf (for various sub-specialty eConsults) frequently found it useful for their situation and would agree to further eConsults [22]. Other studies have cited PCP concerns with eConsults, including an increased workload and dissatisfaction with technology, and specialist concerns of medico-legal liability due to risks of providing advice for a patient not evaluated in-person as well as inadequate protected time for eConsults [10]. Our study did not address these issues specifically; however, PCPs had an opportunity to provide open-ended feedback following an eConsult and we did not receive any feedback regarding technological or any other concerns. In terms of medico-legal liability, the Canadian Medical Protective Association, a national non-profit organization that assists physicians with medical-legal matters and managing risk, has recognized the potential of an eConsultation service to enhance patient care so long as it also serves the patient's needs and supports physicians in meeting their legal, professional and ethical obligations [23]. One advantage of eConsultation from a medico-legal point of view is that it provides documentation of what might otherwise have been an informal conversation between two physicians. The eConsult documentation would be within a Genetics chart if a referral to Genetics was indicated or within a PCP's clinic chart, if no referral was indicated.

eConsultation is among several emerging electronic tools that have the capacity to empower PCPs for the benefit of 


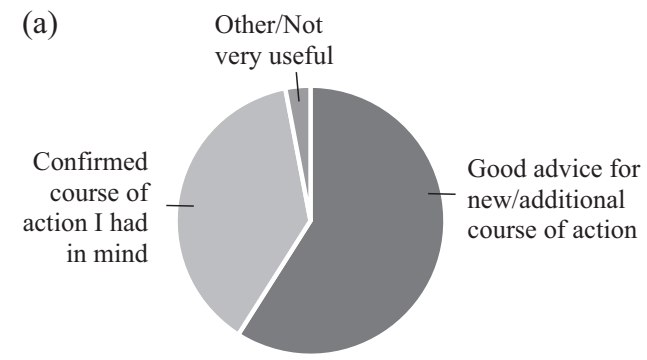

(b)

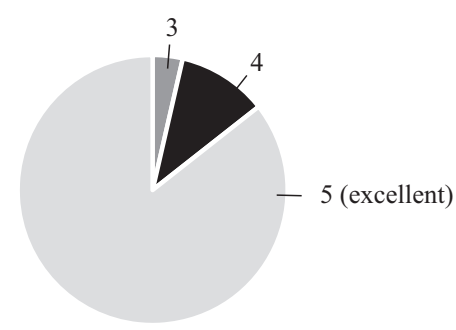

(c)

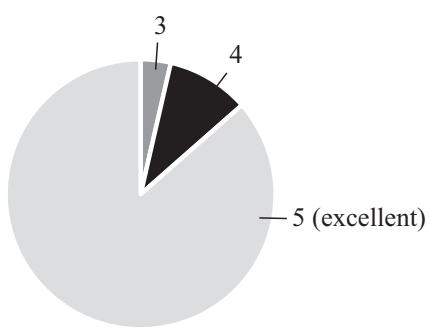

Fig. 1 Primary care provider responses to mandatory close-out survey questions following 111 eConsults. a Which of the following best describes the outcome this eConsult for your patient? b Rate the overall value of the eConsult service in this case for your patient (on a 5-point scale from 1, indicating minimal value, to 5 indicating excellent value). $\mathbf{c}$ Rate the overall value of the eConsult service in this case for you as a primary care provider (on a 5-point scale from 1, indicating minimal value, to 5 indicating excellent value)

patients and the health care system as a whole. Our study suggests that it can improve patient care and optimize health care resources by avoiding unnecessary in-person appointments. It also has the advantage of providing up to date genetics information, which is important given the rapid changes in the field and also the increasing availability of genetic testing to PCPs. As many PCPs work within group practices, the reach of an eConsult in terms of timely sharing of relevant changes in the field and ongoing education may extend beyond an individual PCP to the other members of their practice. To meet the increasing demands for genetics services, the current model of genetics health care delivery must evolve to become more efficient and effective. Integration of an eConsult service creates a stronger and more dynamic link between clinical geneticists and PCPs.

Our study suggests that a clinical genetics eConsultation service is a feasible model for secure communication between a clinical geneticist and PCPs that is of high value to PCPs. This service can provide PCPs with access to a geneticist for clinical support and continuing medical education. With the rising availability of genetic testing to both PCPs and clinical geneticists, and the increasing relevance of genetics care to primary care, this type of communication between geneticists and PCPs allows for optimal patient care. Larger studies with long-term follow-up of a genetics eConsult service will be helpful for implementing genetics eConsult services into clinical practice and transitioning to a more dynamic and efficient clinical genetics model of care.

Acknowledgements This work was supported by the Ministry of Health and Long-Term Care, The Canadian Institutes of Health Research, The Ottawa Hospital Academic Medical Organization Innovation Fund, e-Health Ontario, the University of Ottawa Department of Medicine, Bruyère Research Institute and the Champlain Local Health Integration Network.

\section{Compliance with ethical standards}

Conflict of interest The authors declare that they have no conflict of interest.

Publisher's note: Springer Nature remains neutral with regard to jurisdictional claims in published maps and institutional affiliations.

\section{References}

1. Otten E, Birnie E, Ranchor AV, van Langen IM. Online genetic counseling from the providers' perspective: counselors' evaluations and a time and cost analysis. Eur J Hum Genet. 2016;24:1255-61.

2. Zierhut HA, MacFarlane IM, Ahmed Z, Davies J. Genetic counselors' experiences and interest in telegenetics and remote counseling. J Genet Couns. 2018;27:329-38.

3. Otten E, Birnie E, Lucassen AM, Ranchor AV, Van Langen IM. 2016 Telemedicine uptake among Genetics Professionals in Europe: room for expansion. Eur J Hum Genet. 2016;24:157-63.

4. Kibar Y, Frimberger D, Kropp BP, Reiner W. Accuracy of perinatal diagnosis of $45, \mathrm{X} / 46, \mathrm{XY}$ mosaicism and electronic consultation of affected parents. J Pediatr Urol. 2009;5:274-8.

5. Kentwell M, Dow E, Antill Y, Wrede CD, McNally O, Higgs E et al. Mainstreaming cancer genetics: a model integrating germline $B R C A$ testing into routine ovarian cancer clinics. Gynecol Oncol. 2017;145:130-6.

6. Swanson CL, Kumar A, Maharaj JM, Kemppainen JL, Thomas $\mathrm{BC}$, Weinhold MR et al. Increasing genetic counseling referral rates through bundled interventions after ovarian cancer diagnosis. Gynecol Oncol. 2018;149:121-6.

7. Battista RN, Blancquaert I, Laberge A-M, van Schendel N, Leduc, $\mathrm{N}$. genetics in health care: an overview of current and emerging models. Public Health Genom. 2012;3:34-45.

8. Reiff M, Ross K, Mulchandani S, Propert KJ, Pyeritz RE, Spinner NB et al. Physicians' perspectives on the uncertainties and implications of chromosomal microarray testing of children and families. Clin Genet. 2013;83:23-30.

9. Christensen KD, Vassy JL, Jamal L, Lehmann LS, Slashinski MJ, Perry DL et al. are physicians prepared for whole genome sequencing? a qualitative analysis. Clin Genet. 2016;89:228-34. 
10. Vimalananda VG, Gupte G, Seraj SM, Orlander J, Berlowitz D, Fincke BG et al. Electronic consultations (e-consults) to improve access to specialty care: a systematic review and narrative synthesis. J Telemed Telecare. 2015;21:323-30.

11. Liddy C, Drosinis P, Keely E. Electronic consultation systems: worldwide prevalence and their impact on patient care-a systematic review. J Fam Pract. 2016;33:274-85.

12. Keely E, Liddy C, Afkham A. Utilization, benefits, and implact of an e-consultation service across diverse specialties and primary care providers. Telemed J E Health. 2013;19:733-8.

13. Witherspoon L, Liddy C, Afkham A, Keely E, Mahoney J. Improving access to urologists through an electronic consultation service. Can Urol Assoc. 2017;11:270-4.

14. Lai L, Liddy C, Keely E, Afkham A, Kurzawa J, Abdeen N et al. The impact of electronic consultation on a Canadian tertiary care pediatric specialty referral system: a prospective single-center observational study. PLoS One. 2018;13:1-13.

15. Rostom KC, Smith CD, Liddy C, Afkham A, Keely E. Improving access to rheumatologists: use and benefits of an electronic consultation service. J Rheumatol. 2018;45:137-40.

16. Murthy R, Rose G, Liddy C, Afkham A, Keely E. econsultations to infectious disease specialists: questions asked and impact on primary care providers' behavior. Open Forum Infect Dis. 2017;4:e1-6.
17. Liddy C, Smyth C, Poulin PA, Joschko J, Keely E. Improving access to chronic pain services through consultation: a crosssectional study of the champlain BASE eConsult Service. Pain Med. 2016;17:1049-57.

18. Shehata F, Posner G, Afkham A, Liddy C, Keely E. Evaluation of an electronic consultation service in obstetrics and gynecology in Ontario. Obstet Gynecol. 2016;127:1033-8.

19. Keely E, Liddy C, Epstein G, Afkham A, Williams R, Keller E. Ontario experience with eConsult: moving from pilot to provincial program. Ont Med Rev. 2018; 85:41-3.

20. Liddy C, Rowan MS, Afkham A, Maranger, J, Keely E. Building access to specialist care through e-consultation. Open Med. 2013;7:e1-8.

21. Liddy C, Afkham A, Drosinis P, Joschko J, Keely E. Impact of and satisfaction with a New eConsult Service: a mixed methods study of primary care providers. J Am Board Fam Med. 2015; 28:394-403.

22. Joschko J, Liddy C, Moroz I, Reiche M, Crowe L, Afhkam A et al. Just a click away: exploring patients' perspectives on receiving care through the Champlain $\mathrm{BASE}^{\mathrm{TM}}$ eConsult service. Fam Pract. 2018;35:93-8.

23. Canadian Medical Protective Association (CMPA). Is that eConsultation or eReferral service right for your medical practice? CMPA Perspect Mag. 2017;9:10-3. September 